\title{
Towards a Statistical Atlas of Cardiac Fiber Structure
}

\author{
Jean-Marc Peyrat ${ }^{1}$, Maxime Sermesant ${ }^{1}$, Xavier Pennec ${ }^{1}$, Hervé Delingette $^{1}$, \\ Chenyang $\mathrm{Xu}^{2}$, Elliot McVeigh ${ }^{3}$, and Nicholas Ayache ${ }^{1}$ \\ ${ }^{1}$ INRIA - Asclepios Research Project, Sophia Antipolis, France \\ jean-marc.peyrat@sophia.inria.fr \\ ${ }^{2}$ Siemens Corporate Research, Princeton, New Jersey, USA \\ ${ }^{3}$ Laboratory of Cardiac Energetics, National Heart Lung and Blood Institute, \\ National Institute of Health, Bethesda, Maryland, USA
}

\begin{abstract}
We propose here a framework to build a statistical atlas of diffusion tensors of canine hearts. The anatomical images of seven hearts are first non-rigidly registered in the same reference frame and their associated diffusion tensors are then transformed with a method that preserves the cardiac laminar sheets. In this referential frame, the mean tensor and its covariance matrix are computed based on the Log-Euclidean framework. With this method, we can produce a smooth mean tensor field that is suited for fiber tracking algorithms or the electromechanical modeling of the heart. In addition, by examining the covariance matrix at each voxel it is possible to assess the variability of the cardiac fiber directions and of the orientations of laminar sheets. The results show a strong coherence of the diffusion tensors and the fiber orientations among a population of seven normal canine hearts.
\end{abstract}

\section{Introduction}

While the main geometrical arrangement of myofibers has been known for decades, its variability between subjects and species still remains largely unknown. Understanding this variability is not only important for a better description of physiological principles but also for the planning of patient-specific cardiac therapies 7 . Furthermore, the knowledge of the relation between the myocardium shape and its myofiber architecture is an important and required stage towards the construction of computational models of the heart [14] [1] since the fiber orientation plays a key role when simulating the electrical and mechanical function of the heart. High resolution measurements of fiber orientation has been recently eased with the use of Diffusion Tensor Imaging (DTI) since there is a correlation between the myocardium fiber structure and diffusion tensors [15. DTI also has the advantage to provide directly this information in $3 \mathrm{D}$ with high resolution but unfortunately it is still not available in vivo due to the cardiac motion. There has been several studies [12 13] in the past decade that have measured the variability of fiber orientation from DTI (similar studies has been done for the brain [4]). These studies estimated the fiber direction as 
the primary eigenvector of each tensor and for instance compared its transmural variation with that observed from dissection experiments.

We propose here to extend these studies by building a statistical model of the whole diffusion tensor and not only its first eigenvector. This tensor analysis allows us to study the variability of laminar sheets which are associated with the tertiary eigenvector. Performing this analysis based on vector analysis (instead of tensor analysis) would have been difficult because the secondary and tertiary eigenvalues have often very similar values and may lead to interpretation errors. To the best of our knowledge, this is the first attempt to perform a first and second order statistical analysis of DT images of canine hearts.

Our statistical analysis proceeds as follows. We first register the canine heart images in a common reference frame using anatomical MRIs. For each heart, we get a deformation field we use to register and transform properly the diffusion tensors considering properties of the cardiac fiber microstructure. Finally we use coherent statistical tools on tensors to study the variability of individual hearts from this average model and to evaluate the relevance of such a model. An application of this framework is carried out using a dataset of seven normal canine hearts.

\section{Material and Method}

\subsection{Data Acquisition}

We used a dataset of seven ex vivo fixed normal canine hearts acquired [8] and provided by the Center of Cardiovascular Bioinformatics and Modeling $(\mathrm{CCBM})^{1}$ at the Johns Hopkins University. Each heart was placed in an acrylic container filled with Fomblin, a perfluoropolyether (Ausimon, Thorofare, NJ). Fomblin has a low dielectric effect and minimal MR signal thereby increasing contrast and eliminating unwanted susceptibility artifacts near the boundaries of the heart. The long axis of the hearts was aligned with the z-axis of the scanner. Images were acquired with a 4-element knee phased array coil on a $1.5 T \mathrm{GE}$ $C V / i$ MRI Scanner (GE, Medical System, Wausheka, WI) using a gradient system with $40 \mathrm{mT} / \mathrm{m}$ maximum gradient amplitude and a $150 \mathrm{~T} / \mathrm{m} / \mathrm{s}$ slew rate. Different resolutions have been used around $0.3 \times 0.3 \times 0.9 \mathrm{~mm}^{3}$ and from 14 to 28 gradient directions. The images have been subsampled into $128 \times 128 \times 64$ images with a resolution around $0.6 \times 0.6 \times 1.8 \mathrm{~mm}^{3}$. The temperature during acquisition varied between $18-25^{\circ} \mathrm{C}$ from one heart to another.

\subsection{Myocardium Registration}

In order to analyze the statistical variability of the tensors without introducing a bias, we choose to register the images based on independent information: the anatomical MRIs. Before the registration stage, we pre-process semiautomatically the anatomical MRIs by extracting the image background, and by cropping each image above the valve plane. We register each heart on a

\footnotetext{
${ }^{1}$ http://www.ccbm.jhu.edu/research/DTMRIDS.php
} 
template given by a Procrustes iterative mean estimation. The registration is initialized with an affine global transformation of the hearts defined from three significant landmarks: the apex point and the two corner points of the right ventricle on the valve plane. The apex point is used to define a scaling along the axis of the heart. The matching of the corner points defines a $3 \mathrm{D}$ translation, a $2 \mathrm{D}$ rotation on the valve plane, and a $2 \mathrm{D}$ scaling along a line defined by the 2 corner points. The second registration step is based on a hybrid non-rigid intensity- and landmark-based registration algorithm [2]. This algorithm gives us the ability to interactively refine the registration of a local region. The output of this process is a dense deformation field for each anatomically registered cardiac image.

\subsection{Transformation of Cardiac Diffusion Tensors}

The next stage is to transform the DT image based on the estimated deformation field. For each voxel, the global deformation field is approximated at the first order by an affine transformation [16. This underlying affine transformation $A$ is computed from the identity matrix $I d$ and the Jacobian $\nabla F$ of the deformation field $F: A=I d+\nabla F$.

Now we have the well known problem of applying an affine transformation to a DT image. Since it was shown that there is a correlation between the cardiac tissue microstructure and the eigensystem of the diffusion tensors [8] [13 [15], we can transform the underlying tissue microstructure with the affine transformation. Then we reconstitute the diffusion tensor from this transformed microstructure knowing the relationship between the two of them. The solution we propose here to handle this problem proved to be similar to the Preservation of the Principal Direction (PPD) reorientation strategy proposed in [3]:

$$
V_{1}^{\prime}=\frac{A V_{1}}{\left\|A V_{1}\right\|} \quad V_{3}^{\prime}=\frac{\left(A^{-1}\right)^{T} V_{3}}{\left\|\left(A^{-1}\right)^{T} V_{3}\right\|} \quad V_{2}^{\prime}=V_{3} \otimes V_{1}
$$

where $V_{1}, V_{2}, V_{3}$ are the primary, secondary and tertiary eigenvectors of the original diffusion tensor and where $V_{1}^{\prime}, V_{2}^{\prime}, V_{3}^{\prime}$ are the ones of the transformed diffusion tensor.

The PPD has been already justified and validated in the case of brain DTI 3 . but only partially in the case of cardiac DTI. We propose here a more complete justification of the PPD strategy for cardiac DTI transformation.

As described in Figure 1 the myocardium microstructure is made of laminar sheets of muscle fibers [5]. The space between the laminar sheets is composed of extracellular water and collagen network linking these sheets together. This extracellular space has a mostly unrestricted diffusion in the direction of the two first eigenvectors of the diffusion tensor. The interface plane between the laminar sheets and the extracellular space is an important barrier for water molecules and its normal defines the third eigenvector. The extracellular water between fibers in the laminar planes can explain the difference between the primary eigenvector in the fiber direction and the secondary eigenvector orthogonal to the fiber direction. But the cardiomyocyte geometry is also proposed to explain the privileged diffusion direction in the fiber orientation. A cardiomyocyte is much longer $(50-120 \mu \mathrm{m})$ than wider $(5-25 \mu \mathrm{m})$ and much longer than the mean 
free path (roughly $10 \mu \mathrm{m}$ considering the diffusion time of the acquisitions). It means that the cell membrane does not have as much influence on the water diffusion in the fiber direction as in the other directions where the width of the cells is close to the mean free path.

Let us now analyze the effect of the basic transformations (translations, rotations, shears and scaling) describing an affine transformation. The way to transform the fiber structure and thus the diffusion tensors through translation and rotation is obvious. The scaling only changes the density of fibers microstructure inside a voxel considering the acquired voxel resolution and finally not the diffusion rate. The shearing induced by the affine transformation is not so simple to apply to the diffusion tensors. We illustrate in Figure 1 the shearing applied to the basic microstructure of cardiac fibers. The direct transformation of the original eigenvectors $V_{i}$ leads to the vector $A V_{i}$ and the transformation deduced from the fiber structure deformation leads to the vector $V_{i}^{\prime}$. As we determine the fiber structure deformation through the fiber direction deformation, the transformed primary eigenvector $V_{1}^{\prime}$ is the same as the direct transformation of the original primary eigenvector $V_{1}: V_{1}^{\prime}=\frac{A V_{1}}{\left\|A V_{1}\right\|}$.
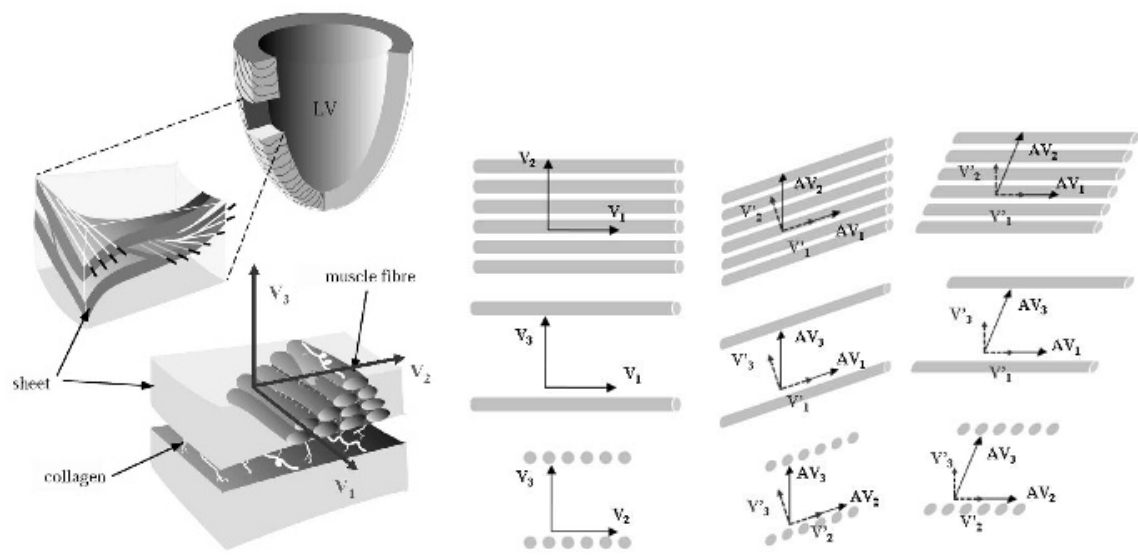

Fig. 1. [Left] Cardiac Fiber Structure (from LeGrice et al. 5]). - [Middle Left] Original basic fiber microstructure with eigenvectors $V_{i}$. [Middle Right and Right] Shearing applied to the basic fiber microstructure : continuous arrows $A V_{i}$ are the transformed eigenvectors through the shearing and dashed arrows $V_{i}^{\prime}$ are the eigenvectors related to the correlation between the fiber microstructure and the diffusion tensor.

The tertiary eigenvector is defined by $n$, the vector normal to the laminar sheets which are considered locally plane. The image of a plane through an affine transformation is a plane. It means that these laminar sheets are stable. We just have to determine $n^{\prime}$ the unit vector normal to the plane defined by the image $V_{1}^{\prime}$ of $V_{1}$ and the image $A V_{2}$ of $V_{2}$ [17]: $n^{\prime}=\frac{\left(A^{-1}\right)^{T} n}{\left\|\left(A^{-1}\right)^{T} n\right\|}$. 
The transformed secondary eigenvector obtained from the correlation between the structure of the fibers happens to be the one that builds an orthonormal basis with the two others. It means that constructing first the secondary (as done for the PPD) or the tertiary (as we do) and then determine the other one to obtain an orthonormal basis leads to the same results. This is the key point that justifies the use of the PPD in our specific case of cardiac DTI.

\subsection{Tensor Statistics}

The Log-Euclidean framework 10 provides a consistent and rigorous framework to study the statistical variability of DTI for each voxel of the heart. In this framework the space of diffusion tensors is a vectorial space which means it inherits from all the statistical properties and tools we can get from a vectorial space. We thus compute the mean of all the registered DTI and the corresponding covariance 11 at each voxel:

$$
\begin{gathered}
\bar{D}_{l o g}=\exp \left(\frac{1}{N} \sum_{i=1}^{N} \log \left(D_{i}\right)\right) \\
\operatorname{Cov}=\frac{1}{N-1} \sum_{i=1}^{N} \operatorname{vect}\left(\Delta D_{i}\right) \cdot \operatorname{vect}\left(\Delta D_{i}\right)^{T}
\end{gathered}
$$

where vect $\left(\Delta D_{i}\right)$ is the vectorial representation [1] of $\Delta D_{i}=\log \left(D_{i}\right)-\log \left(\bar{D}_{\log }\right)$.

The difficulty to visualize the $6 \times 6$ covariance matrix of diffusion tensors leads us to study first its norm $\sqrt{\operatorname{Trace}(\operatorname{Cov})}$, which allows us to identify the variable and stable regions of the heart. To help us in translating the DTI variability into the fiber structure variability, we can analyze this covariance matrix extracting 6 specific variance parameters at each voxel in the coordinate system of the mean tensor: 3 for each eigenvalue variability and the 3 for each pair of orthonormal eigenvectors orientation variability around the third one [6].

\section{Results}

We applied the proposed framework to the dataset of seven canine hearts presented previously. We obtain a smooth cardiac DTI atlas catching the shared transmural variation of the fibers directions (see Figure 2) that is similar to the one generally observed [15. The norm of the covariance matrix in Figure 3 shows a global stability of the compact myocardium and several variable regions especially at the RV and LV endocardial apices where the fiber structure is probably less organized. Some other variabilities at the surface of the heart might also be due to acquisition or registration artifacts.

In order to have a better interpretation of this covariance matrix and to understand the origin of the variabilities, we decompose it into specific modes as described previously. We can see in Figures 3 that the eigenvalues variabilities are homogeneous in the compact myocardium. The mean standard deviations normalized by their mean value $\frac{\bar{\sigma}_{i}}{\lambda_{i}}$ are $0.16,0.24$ and 0.22 for the $1^{\text {st }}, 2^{\text {nd }}$ and $3^{r d}$ eigenvalues. Note that these variabilities and their difference are artificially 


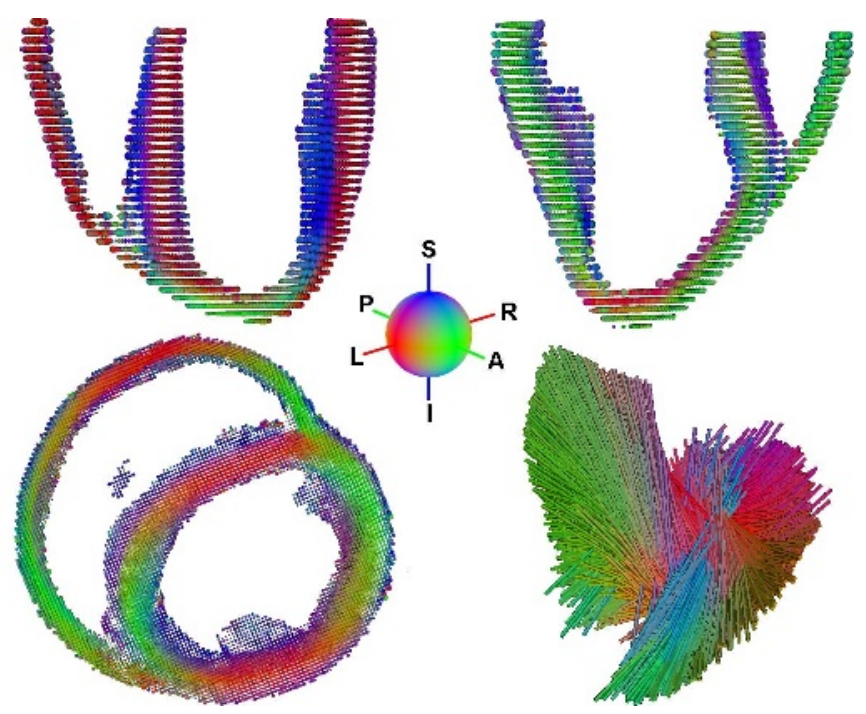

Fig. 2. [Left and Upper Right] Average canine cardiac DTI. [Lower Right] Fiber tracking in the left ventricle wall with high stiffness parameters for a better visualization of the transmural variation of fibers directions. The RGB colors represent the components of the primary eigenvector Red $=\left|V_{x}\right|$, Green $=\left|V_{y}\right|$, Blue $=\left|V_{z}\right|$ with $x, y$ in the axial plane and $z$ orthogonal to the axial plane as described with the colored sphere.
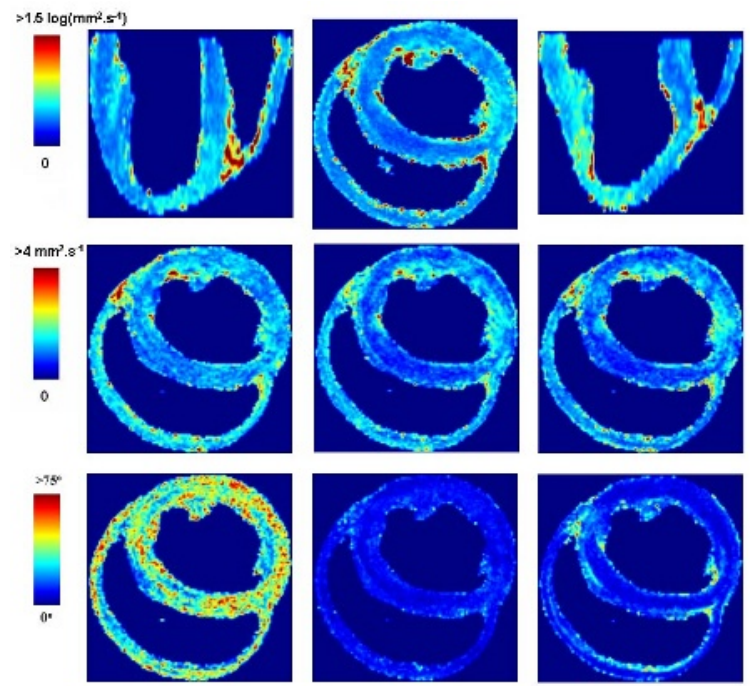

Fig. 3. [upper] Norm of the covariance matrix in 3 orthogonal views. [from middle left to middle right Decomposition of the covariance in 6 eigenmodes describing the variability of the $1^{\text {st }}, 2^{\text {nd }}, 3^{\text {rd }}$ eigenvalues. [from lower left to lower right] Rotation variability of the plane orthogonal to the $1^{\text {st }}, 2^{\text {nd }}$ and $3^{\text {rd }}$ eigenvectors. 
increased by the difference of the temperature during the different image acquisitions (temperature ranging from 18 to $25^{\circ} \mathrm{C}$ ). Anyway mostly the order of the eigenvalues is really meaningful in terms of fiber architecture and thus in terms of electrical conductivity that are introduced in electromechanical modeling (it does not seem to be any direct correlation between the diffusion and electrical conductivity rates). At least these statistical results give us an idea of the average diffusion rates that could help in detecting locally defined pathologies.

The separation of the eigenvalues variability and the eigenvectors orientation variability is important to evaluate the variability of the myocardial fibers architecture. As seen in Figures 3, the orientation of the fibers is stable among a population (mean standard deviation of 8.8 and 9.4 degrees around the secondary and the tertiary eigenvectors) for the two rotations in the planes containing the primary eigenvector. It means that the fiber orientation of the average cardiac DTI is shared by the dataset. The orientation of the laminar sheets described by the rotation of the plane $\operatorname{Span}\left(V_{2}, V_{3}\right)$ around $V_{1}$ shows a much higher mean standard deviation of 23 degrees. First of all this variability is underevaluated considering that the statistical study we proposed is a simplification at the first order [6]. Secondly this variability is due to the difficulty to differentiate the secondary and tertiary eigenvectors when they have similar eigenvalues. When we study the fiber organization (i.e. the 3 rotation eigenmodes) we are not anymore in the diffusion tensor space and an isotropic plane of diffusion leads to a low accuracy in translating it into structural information. Another explanation might also be the existence of two populations of symmetric laminar sheets organization in canine hearts [8] corresponding to the optimal configurations to maximize the systolic shear [9]. Mixing these two populations leads to the computation of an average cardiac fiber architecture model that does not represent a real case of laminar sheets organization. A computation of the normal of the laminar sheets and a further study of their orientation variability is essential to determine its origin and of course to translate an atlas of cardiac DTI into an atlas of the cardiac fiber architecture.

\section{Discussion}

We proposed a theoretically grounded, simple and powerful framework working directly on diffusion tensors to build an average model and to study their variability over a population of cardiac DTIs. The use of these average models instead of analytical models is an important stage to refine the electromechanical modeling with more accurate and reliable data and also with additional informations concerning the anisotropy in the plane orthogonal to the fiber directions.

This framework is a first step towards a statistical atlas of the cardiac fiber architecture that will probably lead to a better understanding of the cardiac fiber architecture shared by a population of healthy or failing hearts, or to compare and to differentiate populations of hearts (canine-human or normal-failing).

In the next step of our research, a larger dataset of hearts would be helpful to get a statistically more reliable atlas. Also, we would like to apply this framework to human hearts in the context of specific clinical applications. 


\section{Acknowledgments}

This research was funded by Siemens Corporate Research, Princeton, NJ. Acquisition of the DTI data was funded by the Intramural Research Program of the National Heart Lung and Blood Institute (E.R. McVeigh Z01-HL4004609). We thank Drs. Patrick A. Helm and Raimond L. Winslow at the Center for Cardiovascular Bioinformatics and Modeling for provision of data, A. Azar for his technical support on the registration tools, P. Fillard for provision of diffusion tensors and fiber tracking computation and visualization tools 2 , and I.J. LeGrice for provision of an illustration in Figure 1.

\section{References}

1. N. Ayache, editor. Computational Models for the Human Body. Handbook of Numerical Analysis. Elsevier, 2004.

2. A. Azar et al. An interactive Intensity- and Feature-Based Non-Rigid Registration Framework for 3D Medical Images. In Proc. of ISBI'06, 2006.

3. D.C. Alexander et al. Spatial Transformations of Diffusion Tensor Magnetic Resonance Images. IEEE TMI, 20(11):1131-1139, 2001.

4. D.K. Jones et al. Spatial normalization and Averaging of Diffusion Tensor MRI Data Sets. NeuroImage, 17:592-617, 2002.

5. I.J. LeGrice et al. Laminar structure of the heart: ventricular myocyte arrangement and connective tissue architecture in the dog. Am J Physiol, 1995.

6. J.M. Peyrat et al. Towards a statistical atlas of cardiac fiber architecture. Research Report 5906, INRIA, May 2006.

7. M. Sermesant et al. Simulation of cardiac pathologies using an electromechanical biventricular model and XMR interventional imaging. Med. Image Anal., 5(9):46780, October 2005.

8. P. Helm et al. Ex Vivo 3D Diffusion Tensor Imaging and Quantification of Cardiac Laminar Structure. Magn. Reson. Med., 54(4):850-859, 2005.

9. T. Arts et al. Relating Myocardial Laminar Architecture to Shear Strain and Muscle Fiber Orientation. Am J Physiol, 280:H2222-H2229, 2001.

10. V. Arsigny et al. Fast and Simple Calculus on Tensors in the Log-Euclidean Framework. In Proc. of MICCAI'05, volume LNCS 3749, pages 115-122, 2005.

11. X. Pennec et al. A Riemannian Framework for Tensor Computing. IJCV, 2006.

12. Y. Cao et al. Large Deformation Diffeomorphic Metric Mapping of Fiber Orientations. In Proc. of ICCV'05, pages 1379-1386, 2005.

13. P. Helm. A Novel Technique for Quantifying Variability of Cardiac Anatomy: Application to the Dyssynchronous Failing Heart. PhD thesis, JHU, 2005.

14. F. Sachse, editor. Computational Cardiology - Modeling of Anatomy, Electrophysiology, and Mechanics. LNCS. Springer, 2004.

15. D.F. Scollan. Reconstructing the Heart: Development and Application of Biophysically-based Electrical Models of Propagation in Ventricular Myocardium Reconstructed from Diffusion Tensor MRI. PhD thesis, JHU, 2002.

16. R. Sierra. Nonrigid Registration of Diffusion Tensor Images. Master's thesis, ETH Zurich, March 2001.

17. K. Turkowski. Graphics Gems, chapter Properties of Surface-Normal Transformations. Academic Press, Inc., 1990.

$\overline{{ }^{2} \text { http://www-sop.inria.fr/asclepios/personnel/Pierre.Fillard/softwares/ }}$ 